\title{
Inpatient Mobility Technicians: One Step Forward?
}

\author{
Daniel L Young, PT, DPT, PhD 1,3; Daniel J Brotman, MD²; Erik H Hoyer, MD,2*
}

'Department of Physical Medicine and Rehabilitation, Johns Hopkins University, Baltimore, Maryland; ${ }^{2}$ Division of General Internal Medicine, Johns Hopkins University, Baltimore, Maryland; ${ }^{3}$ Department of Physical Therapy, University of Nevada Las Vegas, Las Vegas, Nevada.

rolonged bedrest with minimum mobility is associated with worse outcomes for hospitalized patients, particularly the elderly. ${ }^{1,2}$ Immobility accelerates loss of independent function and leads to complications such as deep vein thrombosis, pressure ulcers, and even death.,4 Increasing activity and mobility early in hospitalization, even among critically ill patients, has proven safe. ${ }^{5}$ Patients with intravascular devices, urinary catheters, and even those requiring mechanical ventilation or extracorporeal membranous oxygenation can safely perform exercise and out-of-bed activities. $^{5}$

Although the remedy for immobility and bedrest seems obvious, implementing workflows and strategies to increase inpatient mobility has proven challenging. Physical therapistsoften the first solution considered to mobilize patients-are a limited resource and are often coordinating with other team members on care planning activities such as facilitating discharge, arranging for equipment, and educating patients and families, rather than assisting with routine mobility needs. ${ }^{6}$ Nurses share responsibility for patient activity, but they also have broad patient-care responsibilities competing for their time. ${ }^{7}$ Additionally, some nurses may feel they do not have the necessary training to safely mobilize patients. ${ }^{8,9}$

In this context, the work by Rothberg et al. is a welcome addition to the literature..$^{10}$ In this single-blind randomized pilot trial, 102 inpatients aged 60 years and older were randomly assigned to either of two groups: intervention (ambulation protocol) or usual care. In the intervention arm, dedicated mobility technicians-ie, redeployed patient-care nursing assistants trained in safe patient-handling practices-were tasked to help patients walk three times daily. Patients in the intervention group took significantly more steps on average compared with those receiving usual care (994 versus 668). Additionally, patients with greater exposure to the mobility technicians ( $>2$ days) had significantly higher step counts and were more likely to achieve $>900$ steps per day, below which patients are likely to experience functional decline. ${ }^{11}$ This study highlights the feasibility of using trained mobility technicians rather than

*Corresponding Author: Erik H Hoyer, MD; E-mail: ehoyer1@jhmi.edu; Telephone: 410-502-2441; Twitter: @HopkinsAMP

Published online first February 20, 2019

Received: November 28, 2018; Revised: December 19, 2019;

Accepted: December 19, 2019

๑ 2019 Society of Hospital Medicine DOI 10.12788/jhm.3148 more expensive providers (eg, physical therapists, occupational therapists, or nurses) to enhance inpatient ambulation.

The authors confirmed previously known findings that inpatient mobility, which was assessed in this study by accelerometers, predicts post-hospital patient disposition. Although consumer grade accelerometer devices (eg, Fitbit ${ }^{\oplus}$ ), have limitations and may not count steps accurately for hospitalized patients who walk slowly or have gait abnormalities, ${ }^{12}$ Rothberg et al. still found that higher step count was associated with discharge home rather than to a facility. Discharge planning in the hospital is often delayed because clinicians fail to recognize impaired mobility until after resolution of acute medical/surgical issues. ${ }^{13}$ The use of routinely collected mobility measurements, such as step count, to inform decisions around care coordination and discharge planning may ultimately prove helpful for hospital throughput.

Despite the increased mobility observed in the intervention group, discharge disposition after hospitalization and hospital length of stay (LOS) did not differ between groups, whether analyzed according to per-protocol or intention-to-treat analysis. Although LOS and discharge disposition are known to be associated with patient functional status, they are also influenced by other factors, such as social support, health insurance, medical status, and patient or family preferences. ${ }^{14-17}$ Furthermore, illness severity may confound the association between step count and outcomes: sicker patients walk less, stay longer, and are more likely to need postacute rehabilitation. Thus, the effect size of a mobility intervention may be smaller than expected based on observational data, leading to underpowering. Another possibility is that the intervention did not affect these clinical outcomes because patients in the intervention group only received the intervention for an average of one-third of their hospitalization period and the mobility goal of three times per day was not consistently achieved. Mobility technician involvement was often delayed because the study required physical therapy evaluations to determine patient appropriateness before the mobility intervention was initiated. This aspect of study design belies a commonplace cultural practice to defer inpatient mobilization until a physical therapist has first evaluated the patient. Moreover, limiting mobility interventions to a single provider, such as a mobility technician, can mean that patients are less likely to be mobilized if that resource is not available. Establishing an interdisciplinary culture of mobility is more likely to be successful. ${ }^{18}$ One possible strategy is to start with nurse-performed systematic assessments of functional ability to set daily mobility goals that any appro- 
priate provider, including a mobility technician, could help to implement. ${ }^{19,20}$

Although studies designed to increase hospital mobility have yielded mixed results, ${ }^{21}$ and larger high-quality clinical trials are needed to demonstrate clear and consistent benefits on patient-centered and operational outcomes, we applaud research and quality improvement efforts (including the current study) that promote inpatient mobility through strategies and measurements that do not require intensive physical therapist involvement. Mobility technicians may represent one step forward in enhancing a culture of mobility.

Disclosures: The authors certify that no party having a direct interest in the results of the research supporting this article has or will confer a benefit on us or on any organization with which we are associated.

\section{References}

1. Brown CJ, Redden DT, Flood KL, Allman RM. The underrecognized epidemic of low mobility during hospitalization of older adults. J Am Geriatr Soc. 2009;57(9):1660-1665. doi:10.1111/j.1532-5415.2009.02393.x

2. Greysen SR. Activating hospitalized older patients to confront the epidemic of low mobility. JAMA Intern Med. 2016;176(7):928. doi:10.1001/jamainternmed.2016.1874

3. Covinsky KE, Pierluissi E, Johnston CB. Hospitalization-associated disability: "she was probably able to ambulate, but I'm not sure". JAMA. 2011;306(16):1782-1793. doi:10.1001/jama.2011.1556

4. Wu X, Li Z, Cao J, et al. The association between major complications of immobility during hospitalization and quality of life among bedridden patients: a 3 month prospective multi-center study. PLOS ONE. 2018;13(10):e0205729. doi:10.1371/journal.pone.0205729

5. Nydahl P, Sricharoenchai T, Chandra S, et al. Safety of patient mobilization and rehabilitation in the intensive care unit: systematic review with meta-analysis. Ann Am Thorac Soc. 2017;14(5):766-777. doi:10.1513/AnnalsATS.201611-843SR

6. Masley PM, Havrilko C-L, Mahnensmith MR, Aubert M, Jette DU, Coffin-Zadai C. Physical Therapist practice in the acute care setting: a qualitative study. Phys Ther. 2011;91(6):906-922. doi:10.2522/ptj.20100296

7. Young DL, Seltzer J, Glover M, et al. Identifying barriers to nurse-facilitated patient mobility in the intensive care unit. Am J Crit Care Off Publ Am Assoc Crit-Care Nurses. 2018;27(3):186-193. doi:10.4037/ajcc2018368

8. Brown CJ, Williams BR, Woodby LL, Davis LL, Allman RM. Barriers to mobility during hospitalization from the perspectives of older patients and their nurses and physicians. J Hosp Med Off Publ Soc Hosp Med. 2007;2(5):305-313. doi:10.1002/jhm.209

9. Hoyer EH, Brotman DJ, Chan KS, Needham DM. Barriers to early mobility of hospitalized general medicine patients: survey development and results. Am J Phys Med Rehabil. 2015;94(4):304-312. doi:10.1097/ PHM. 0000000000000185

10. Hamilton AC, Lee N, Stilphen M, et al. Increasing Mobility via In-hospital Ambulation Protocol Delivered by Mobility Technicians: A Pilot Randomized Controlled Trial. J Hosp Med. 2019;14(4):272-277. doi: 10.12788/jhm.3152.

11. Agmon M, Zisberg A, Gil E, Rand D, Gur-Yaish N, Azriel M. Association Between 900 Steps a Day and Functional Decline in Older Hospitalized Patients. JAMA Intern Med. 2017;177(2):272. doi:10.1001/jamainternmed.2016.7266

12. Anderson JL, Green AJ, Yoward LS, Hall HK. Validity and reliability of accelerometry in identification of lying, sitting, standing or purposeful activity in adult hospital inpatients recovering from acute or critical illness: a systematic review. Clin Rehabil. 2018;32(2):233-242. doi:10.1177/0269215517724850

13. Roberts DE, Holloway RG, George BP. Post-acute care discharge delays for neurology inpatients: Opportunity to improve patient flow. Neurol Clin Pract. July 2018:8(4):302-310. doi:10.1212/CPJ.0000000000000492

14. Hoyer EH, Friedman M, Lavezza A, et al. Promoting mobility and reducing length of stay in hospitalized general medicine patients: A quality-improvement project. J Hosp Med. 2016;11(5):341-347. doi:10.1002/jhm.2546

15. Surkan MJ, Gibson W. Interventions to mobilize elderly patients and reduce length of hospital stay. Can J Cardiol. 2018;34(7):881-888. doi:10.1016/j. cjca.2018.04.033

16. Ota H, Kawai H, Sato M, Ito K, Fujishima S, Suzuki H. Effect of early mobilization on discharge disposition of mechanically ventilated patients. J Phys Ther Sci. 2015;27(3):859-864. doi:10.1589/jpts.27.859

17. Hoyer EH, Young DL, Friedman LA, et al. Routine inpatient mobility assessment and hospital discharge planning. JAMA Intern Med. 2018. doi:10.1001/ jamainternmed.2018.5145

18. Czaplijski T, Marshburn D, Hobbs T, Bankard S, Bennett W. Creating a culture of mobility: an interdisciplinary approach for hospitalized patients. Hosp Top. 2014;92(3):74-79. doi:10.1080/00185868.2014.937971

19. Hoyer EH, Young DL, Klein LM, et al. Toward a common language for measuring patient mobility in the hospital: reliability and construct validity of interprofessional mobility measures. Phys Ther. 2018;98(2):133-142.. doi:10.1093/ $\mathrm{ptj} / \mathrm{pzx} 110$

20. Klein $L M$, Young $D$, Feng $D$, et al. Increasing patient mobility through an individualized goal-centered hospital mobility program: a quasi-experimental quality improvement project. Nurs Outlook. 2018;66(3):254-262. doi:10.1016/j.outlook.2018.02.006

21. Kanach FA, Pastva AM, Hall KS, Pavon JM, Morey MC. Effects of structured exercise interventions for older adults hospitalized with acute medical illness: a systematic review. J Aging Phys Act. 2018;26(2):284-303. doi:10.1123/ japa.2016-0372 